\title{
Experimental Investigation of Superdiffusion via Coherent Disordered Quantum Walks
}

\author{
Andrea Geraldiø ${ }^{1,}{ }^{, *}$ Alessandro Laneve, ${ }^{1}$ Luis Diego Bonavena, ${ }^{1}$ Linda Sansoni, ${ }^{1}$ Jose Ferraz $\odot,{ }^{2}$ \\ Andrea Fratalocchi, ${ }^{3}$ Fabio Sciarrino, ${ }^{1}$ Álvaro Cuevas, ${ }^{1,4}$ and Paolo Mataloni ${ }^{1}$ \\ ${ }^{1}$ Dipartimento di Fisica, Sapienza Università di Roma, Piazzale Aldo Moro, 5, I-00185 Roma, Italy \\ ${ }^{2}$ Departamento de Física, Universidade Federal Rural de Pernambuco, 52171-900 Recife, Brazil \\ ${ }^{3}$ PRIMALIGHT, Faculty of Electrical Engineering; Applied Mathematics and Computational Science, \\ King Abdullah University of Science and Technology (KAUST), Thuwal 23955-6900, Saudi Arabia \\ ${ }^{4}$ ICFO-Institut de Ciències Fotòniques, The Barcelona Institute of Science and Technology, \\ 08860 Castelldefels, Barcelona, Spain
}

(Received 26 July 2019; published 1 October 2019)

\begin{abstract}
Many disordered systems show a superdiffusive dynamics, intermediate between the diffusive one, typical of a classical stochastic process, and the so-called ballistic behavior, which is generally expected for the spreading in a quantum process. We have experimentally investigated the superdiffusive behavior of a quantum walk, whose dynamics can be related to energy transport phenomena, with a resolution which is high enough to clearly distinguish between different disorder regimes. By our experimental setup, the region between ballistic and diffusive spreading can be effectively scanned by suitably setting few degrees of freedom and without applying any decoherence to the quantum walk evolution.
\end{abstract}

DOI: 10.1103/PhysRevLett.123.140501

Introduction.-The study of energy transport phenomena in heterogeneous systems, like synthetic or biological media, ranging from tissues to computation nodes, enlightens the role of quantum coherence [1,2] which is believed to enhance the rate of these processes [3,4]. In such conditions, a superdiffusive dynamic settles in the transport or propagation processes. This is the case of heat excitation transport in particular condensed matter systems such as one dimensional (1D) metal lattices, described by the Luttinger liquids theory [5]. Studies performed in this context have shown a violation in the dichotomy between ballistic and diffusive transport regimes. There are several cases in which superdiffusion occurs, for example in classical one-dimensional systems [6], when both disorder and nonlinear effects are present [7], and even in quantum systems experiencing many-body localization phase transitions [8].

Quantum walks (QWs) have long been found to efficiently describe coherent energy transport [3,9]. By suitably introducing disorder in a QW, it is possible to modify its spreading behavior. A paradigmatic example is given by Anderson localization [10], originally formulated for condensed matter systems, and extensively demonstrated in the case of all-optical systems [11-13]. The theoretical and experimental studies carried out on this topic have covered a wide range of phenomena, including "hypertransport" of light $[14,15]$, and disordered QWs preserving the time dependence of spreading [16], and decoherence effects determining the transition from quantum to classical random walks (CRWs) $[15,17]$. In these studies the role of disorder source was played by decoherence or various types of unitary evolutions. The present work deals with the description of the transition between quantum and classical regimes, while the coherence of the evolution is preserved. This has been done experimentally by implementing proper configurations of disorder in a discrete quantum walk $(\mathrm{QW})$.

Theoretical model.-The theoretical description of a discrete QW is based on a walker, a coin and the operators acting on them $[18,19]$. In a $1 \mathrm{D} \mathrm{QW}$, the walker is given by a quantum particle which is in a superposition of the position states, described by the set of states $\left\{|i\rangle_{p}\right\}$, each of them corresponding to a particular site of the line. The coin is represented by any internal binary degree of freedom of the particle, given by the basis kets $\left\{|0\rangle_{c},|1\rangle_{c}\right\}$. The evolution of the quantum particle is controlled by the coin operator $\hat{C}$, acting on the coin state, and by the shift operator $\hat{S}$, which moves the walker according to the coin state. The evolution of the walker is then described by the repeated action of coin and shift operators on the general state of the particle $|\psi\rangle=\sum_{i, j} P_{i, j}|i\rangle_{p} \otimes|j\rangle_{c},\left|\psi^{\prime}\right\rangle=\hat{S}$. $(\hat{I} \otimes \hat{C})|\psi\rangle$ where the coin operator is expressed as $\hat{C}=$ $(1 / \sqrt{2})\left(|0\rangle_{c}\left\langle\left. 0\right|_{c}+\mid 0\right\rangle_{c}\left\langle\left. 1\right|_{c}+\mid 1\right\rangle_{c}\left\langle\left. 0\right|_{c}-\mid 1\right\rangle_{c}\left\langle\left. 1\right|_{c}\right)\right.$ while the shift operator reads $\hat{S}=\sum_{i}|i-1\rangle_{p}\left\langle\left. i\right|_{p} \otimes \mid 0\right\rangle_{c}\left\langle\left. 0\right|_{c}+\right.$ $|i+1\rangle_{p}\left\langle\left. i\right|_{p} \otimes \mid 1\right\rangle_{c}\left\langle\left. 1\right|_{c}\right.$. These equations describe the evolution of a walker in a completely ordered QW, in which the coin operator is uniform both in space and time. Other structures of the coin operator, based on suitable maps of the QW phases, are possible [20].

Here, by randomly shifting the phase difference imposed by the coin operator, we are able to simulate the superdiffusive behavior of a quantum walker and to investigate 
the transition from the ballistic regime of an ordered QW to the diffusive one of a CRW.

The operator describing the phase shift at a certain step $n$ of the evolution can be written as $\hat{P}_{n}=\sum_{i}|i\rangle_{p}\left\langle\left. i\right|_{p} \otimes\right.$ $\left(e^{i \phi_{|0\rangle}^{i}(n)}|0\rangle_{c}\left\langle\left. 0\right|_{c}+e^{i \phi_{|1\rangle}^{i}(n)} \mid 1\right\rangle_{c}\left\langle\left. 1\right|_{c}\right)\right.$, where $\phi_{|0\rangle}^{i}(n)\left(\phi_{|1\rangle}^{i}(n)\right)$ corresponds to the phase shift imposed by the coin operator in the site $i$ to the state $|0\rangle_{c}\left(|1\rangle_{c}\right)$. We define the degree of disorder $p$ as the percentage of random phases that the walker experiences during the evolution. As a consequence, the case $p=0$ corresponds to a standard ordered QW while for $p=1$ the QW becomes completely disordered (case of a CRW). We call this kind of quantum walk $p$ diluted $\mathrm{QW}$, because of the dilution of a given degree of disorder $p$ during the $\mathrm{QW}$ evolution. With no further assumptions this model describes a completely spaceand time-uncorrelated disorder.

We limit our analysis to the case in which the phases $\phi_{|0\rangle}^{i}(n)$ and $\phi_{|1\rangle}^{i}(n)$ can only be 0 or $\pi$. We are interested to study how the quantum to classical transition depends on the parameter $p$. For a given value of $p$ a number of different phase maps can be realized, in principle, corresponding to different evolutions of the probability distribution. Thus, the values of the relevant parameters identifying a given value of $p$ are obtained by averaging over many different phase map configurations characterized by the same degree of disorder.

A useful quantity in the study of the single particle evolution is the variance of the position probability distribution, defined as

$$
\operatorname{Var}_{1}(n)=\sum_{i=-n}^{n} i^{2} P_{i}(n)-\left[\sum_{i=-n}^{n} i P_{i}(n)\right]^{2},
$$

where $P_{i}(n)$ is the probability to find the walker on site $i$ at step $n$. We simulated the behavior of the variance for different values of $p$ up to 20 steps, in the range $p=0$ to 1 , with a progressive increment of 0.01 . For each value of $p$ we simulated the evolution of 1000 different phase maps, then we computed the mean value of the variance. In order to show how good this model of disorder is to study the superdiffusion, we computed the standard deviation of the variance distribution for each value of $p$. Results of simulation for 7 and 20 steps are shown in Fig. 1. In both cases the mean value of variance decreases with increasing of $p$, since a constant value around $p \sim 0.40$ is approached for both 7 and 20 steps. This indicates that the output distribution rapidly merges in a classical one for $p>0.40$, while a genuine superdiffusive behavior occurs between $0<p \leq 0.20$. Focusing on this region we observe that, for a given value of $\operatorname{Var}_{1}(p)$, the uncertainty on the corresponding value of $p$ decreases with increasing of $n$.

A further useful quantity is the similarity which is a measure of how two distributions $G$ and $G^{\prime}$ are similar: $S\left(G, G^{\prime}\right)=\left[\left(\sum_{i} \sqrt{G_{i} G_{i}^{\prime}}\right)^{2} / \sum_{i} G_{i} \sum_{j} G_{j}^{\prime}\right]$. In the graph of

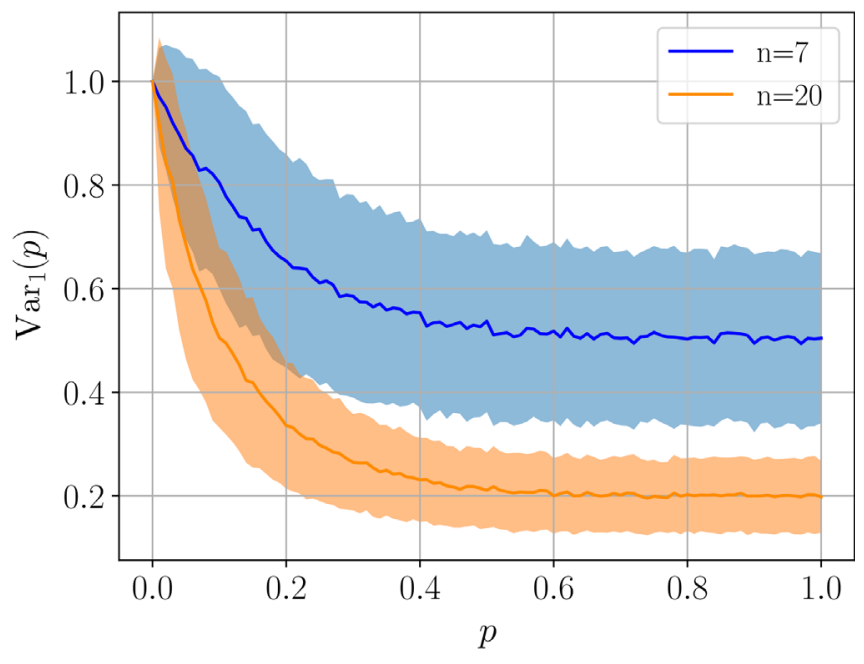

FIG. 1. Simulation of the variance behavior for 7 and 20 steps as a function of $p$. The average values of the variance have been calculated on a sample of 1000 different phase maps for each value of $p$. In the vertical axes $\operatorname{Var}_{1}(p)$ for 7 and 20 steps have been normalized to their maximum values. Error bands correspond to the standard deviation of the variance distributions.

Fig. 2 we show the behavior of the similarity between the average distribution for a given value of disorder $p$ and the ordered or disordered distribution at varying of $p$ (respectively, solid and dashed lines). The similarity with the ordered (disordered) distribution decreases (increases) with $p$ since the higher the disorder the more destructive interference tend to reproduce the disordered distribution.

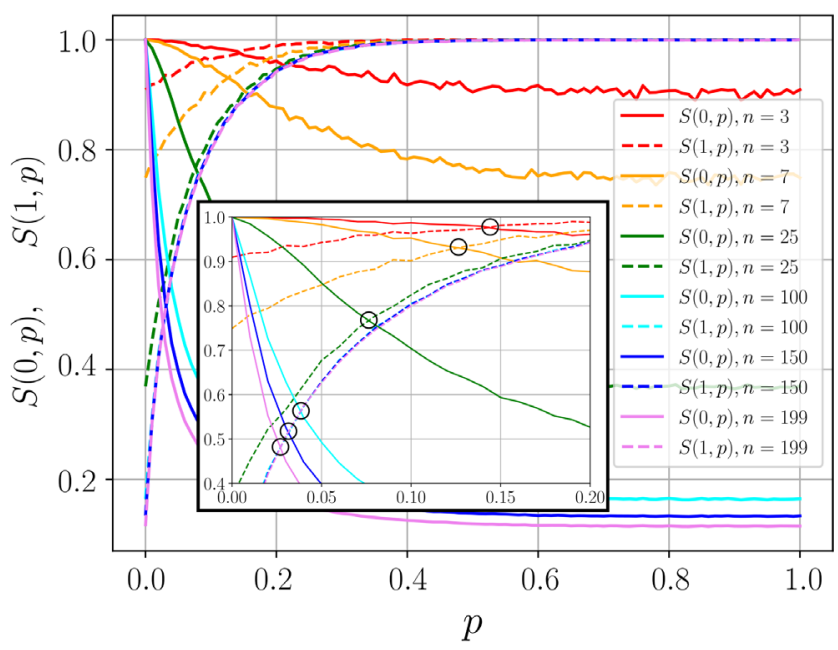

FIG. 2. Computational simulations for similarity between ordered (solid line) and disordered (dashed line) distributions [respectively, $G(p=0) \equiv G(0)$ and $G(p=1) \equiv G(1)]$ and the average distribution for a given value $p$ as a function of $p$. Here, $S(0, p)[S(1, p)]$ stands for $S[G(0), G(p)]\{S[G(1), G(p)]\}$. In the inset a zoom on the intersection region is reported. The crossing point shifts towards lower values of $p$ when the step number increases. Different colors stand for different step numbers of the evolution. 
In the inset, the crossing points between the two quantities identify the transition between quantum and classical regimes for a given number of step $n$. It is worth noting that the transition point decreases with $n$. This continuous transition occurs without loosing completely the quantum features of the evolution and can be interpreted as an evidence of the fact that the QW modifies its behavior because of the total amount of disorder experienced along its spreading.

Experimental implementation.-A 1D quantum walk can be realized by a network of beam splitters (BSs), each of them representing a particular site of the line and acting both as $\hat{C}$ and $\hat{S}$ operators. Several implementations of optical QW have been realized, based on bulk optics schemes, bulk-fiber circuits and femtosecond laser written photonic circuits [13,21-24]. The bulk optic setup used in the present work consists of two displaced multipass Sagnac interferometers (SIs) linked by a common beam splitter $\left(\mathrm{BS}_{1}\right.$ in Fig. 3).

Beam displacement is obtained by translating one mirror of $\mathrm{SI}_{1}$ along the $x$ direction. This particular configuration allows us to create in the $x-y$ horizontal plane of Fig. 3 a (quasi-)infinite loop, which is equivalent to a chain of phase-stable and independently tunable Mach-Zehnder interferometers [25]. The entire BS network necessary for a QW is realized by exploiting also the $z$ direction. On this purpose, suitable beam displacers (BDs) are inserted along clockwise directions in $\mathrm{SI}_{1}$ and counterclockwise ones in $\mathrm{SI}_{2}$ allowing us to increase the number of $x-y$ planes on which the photons can travel [see Supplemental Material [26] (SM)]. Phases are independently addressed in each unit mesh of the QW by using rotating glass plates (RP). The output modes of each step can be extracted for measurement by a set of moving

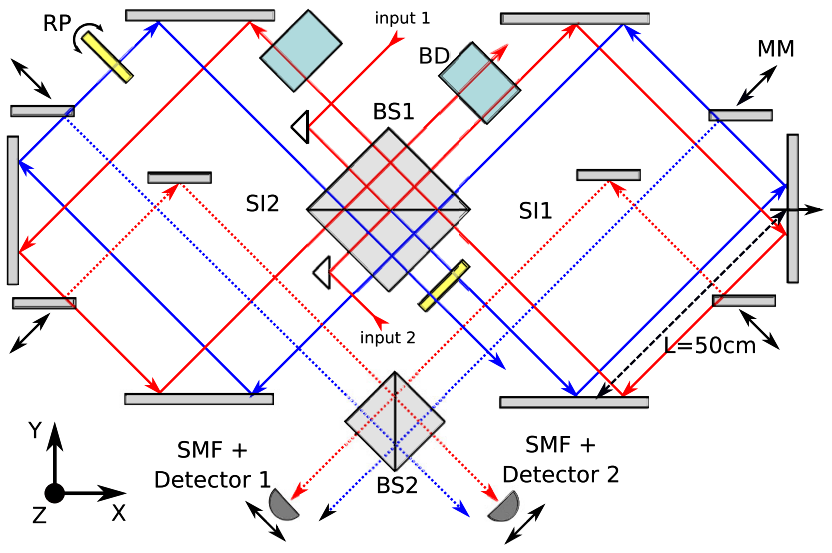

FIG. 3. Sketch of the setup used in the experiment. BS, beam splitter; BD, beam displacer; RP, rotating glass plates; MM, moving mirror; SMF, single mode fiber. Each side of the square SI is $50 \mathrm{~cm}$ long. Blue and red beams circulate in opposite directions and impinge on the $\mathrm{BS}_{1}$ in the same horizontal point but at different heights along the $z$ direction, due to the effect of BD. mirrors (MMs), intersecting and extracting from the setup only the modes at the selected step $N_{j}$, without intersecting the steps $N<N_{j}$. The extracted radiation is then coupled on a single mode fiber and measured (for further details on the setup see [25] and [27]). A slight modification of the setup allows us to inject two distinguishable or indistinguishable photons through the two different input ports of $\mathrm{BS}_{1}$. After the extraction of the modes, a further $\mathrm{BS}\left(\mathrm{BS}_{2}\right)$ allows us to separate photons traveling along the same mode. Adding a further coupler intercepting the extracted modes, coincidences between all possible modes at a given step can be measured and the whole two photon probability distribution can be experimentally reconstructed (see SM [26] for further details).

Experimental results.- It is well known that in a superdiffusive process the spread of the walker position follows a power law, expressed in our case by $\operatorname{Var}_{1}(n) \propto n^{\beta}$, with $n$ corresponding to the number of steps and $1<\beta<2$ [28]. Here, the case $\beta=2(\beta=1)$ is typical of a QW (CRW). We experimentally implemented five different values of $p$, namely $0,0.05,0.10,0.20$, and 1 . For each value of $p>0$ we selected three different phase maps and experimentally reconstructed the output probability distribution for each step of the evolution. In Fig. 4 the average variance of the walker is reported as a function of the step number $n$.

The experimental setup allows us to start the QW only from the central position of the 1D lattice and by using

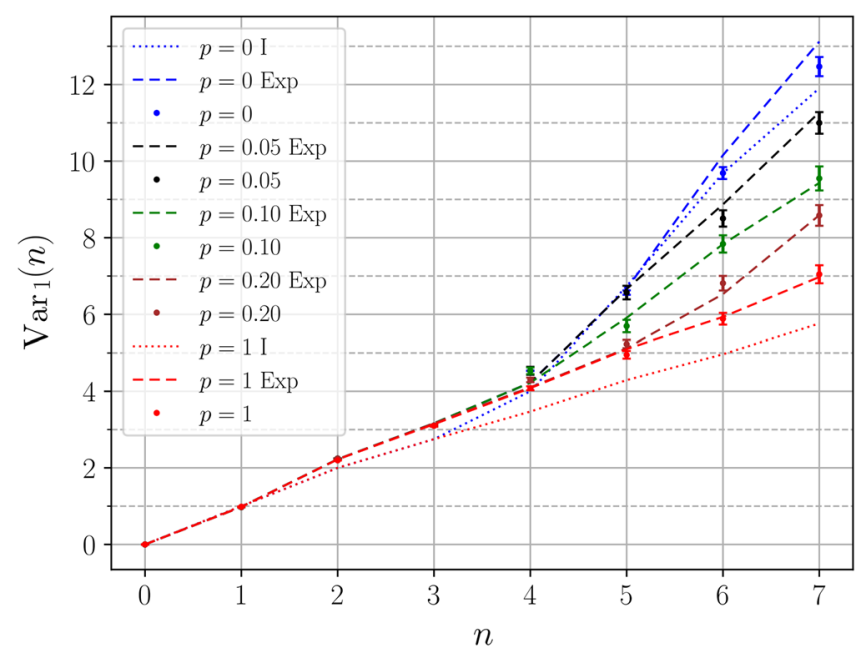

FIG. 4. Experimental values of the variance as a function of the number of steps for different values of $p(0,0.05,0.1,0.2,1)$. Dots correspond to experimental data. For a given value of $p$, the dashed line shows the expected average behavior (Exp) of variance for the three selected phase maps. They were computed by taking into account the actual optical parameters of the setup. Dotted lines show the ideal behavior $(I)$ of a perfectly symmetric $\mathrm{BS}_{1}$ in the two limit cases, namely the ordered and disordered QW. Note that, up to the third step, the variance is not affected by the phase thus the red and blue dotted lines are coincident; the same effect occurs for all the dashed lines. 
TABLE I. Expected $\left(\beta_{\text {theo }}\right)$ and experimental $\left(\beta_{\text {fit }}\right)$ values of the exponent $\beta$ for different values of $p$. The values between 1 and 2 for $p>0$ confirm the superdiffusive behavior of the evolution.

\begin{tabular}{llc}
\hline \hline$p$ & $\beta_{\text {theo }}$ & $\beta_{\text {fit }}$ \\
\hline 0 & 1.69 & $1.64 \pm 0.10$ \\
0.05 & 1.540 & $1.433 \pm 0.067$ \\
0.10 & 1.414 & $1.277 \pm 0.061$ \\
0.20 & 1.198 & $1.160 \pm 0.060$ \\
1 & 0.921 & $0.961 \pm 0.022$ \\
\hline \hline
\end{tabular}

any of the two coin modes (input ports of $\mathrm{BS}_{1}$ ). Accordingly, we studied the evolution for the input state $|\psi\rangle=|0\rangle_{p} \otimes|0\rangle_{c}$. Experimental data are in excellent agreement with the theoretical prediction for the three chosen phase maps (dashed lines). The whole superdiffusive region, namely the one between the diffusive (red lines) and the ballistic (blue lines) behaviors, can be exploited by varying the values of $p$. A small amount of phase instability was present due to the large size of the apparatus. This effect has been included in the computation of errors bars. We also show in Fig. 4 the expected behaviors simulated by considering ideal optical elements for the two limit cases of ordered and completely disordered QW (respectively, blue and red dotted lines). They differ from the experimental data because of the nonperfectly symmetric behavior of $\mathrm{BS}_{1}$ (see $\mathrm{SM}$ for details [26]) [27].

The actual superdiffusive behavior of the experimental evolutions is shown in Table I, where we report the values of $\beta$ obtained by fitting the experimental data $\left(\beta_{\mathrm{fit}}\right)$ and comparing them with the values obtained through theoretical simulations $\left(\beta_{\text {theo }}\right)$. The observed discrepancies can be explained as follows.

(i) The $\beta_{\text {theo }}$ values have been obtained without considering the experimental components imperfections, e.g., BS asymmetry and losses.

(ii) Numerical simulations for $p>0$ have been obtained, as said, by averaging over 1000 disorder configurations, while three phase maps were used in the experiment. Thus, we expect the simulation results to be far more accurate, while the experimental ones are more sensitive to stochastic deviations.

It is worth noting that, for the ordered case, the values of $\beta_{\text {theo }}$ and $\beta_{\text {fit }}$ are lower than the expected value of 2 . Indeed the quadratic growth is an asymptotic behavior, expected to be achieved for long evolution times. We can conclude that a superdiffusive behavior, uniquely identified by the condition $1<\beta<2$, can be simulated using $p$-diluted QWs, making them a useful tool for further investigations on diffusion processes. We also investigated the case of indistinguishable photons entering the QW through the two $\mathrm{BS}_{1}$ 's input ports (see Fig. 3). The variance of the mean position of two indistinguishable walkers, whose expression is

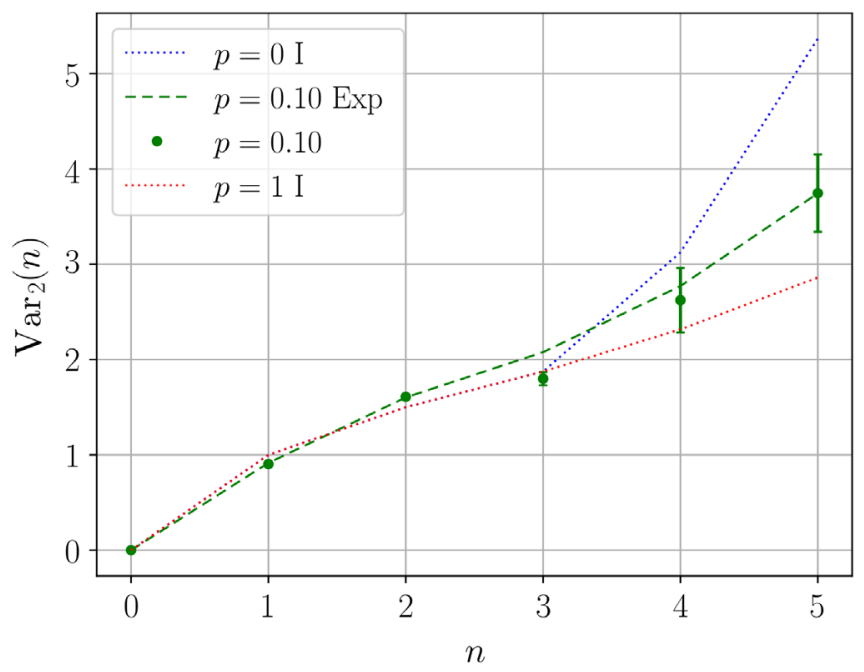

FIG. 5. Experimental results for the two photon QW. Dots represent experimental data, dashed lines indicate the expected behavior obtained by taking into account the real parameters of the setup (Exp), dotted lines correspond to the limit cases of the ideal ordered and disordered two photon QW (I). Note that, up to the third step, the variance is not affected by the phase thus the red and blue dotted lines are coincident.

$\operatorname{Var}_{2}(n)=\sum_{i, j=-n}^{n}\left(\frac{i+j}{2}\right)^{2} P_{i, j}(n)-\left(\sum_{i, j=-n}^{n} \frac{i+j}{2} P_{i, j}(n)\right)^{2}$

is given in Fig. 5 for $p=0.10$. In this case the number of steps is limited to $n=5$ mostly for two reasons: the losses present in the apparatus, limiting the number of detectable coincidences, and the growth of the number of modes on which the photons can travel. Experimental data are in very good agreement with theoretical simulation taking into account the real setup parameters. Simulations of the behavior of $\mathrm{Var}_{2}$ in the ordered and disordered case are also reported. Error bars are larger than the ones in the single photon case due to the fact that in this case a little variation of the phase must be considered twice, because it is experienced by both photons (see SM [26] for further details on the two photon case).

Conclusion.-We have experimentally investigated the superdiffusion process, intermediate between the quantum and classical transport regimes, by introducing tunable disorder, through suitable phase map configurations, in a discrete QW. The intrinsically stable bulk optic system used in the experiment allows us to operate with any kind of phase map. By increasing the number of steps exploited by the optical setup, the subdiffusive regime, localized between the diffusive and the localized one, can also be experimentally explored. The $p$-diluted QW approach described in this work makes possible to simulate a decoherent process without altering the coherent evolution 
of the walker. We are currently investigating on the relationship existing between the two models.

We acknowledge support from the European Commission Grants No. FP7-ICT-2011-9-600838 (QWAD-Quantum Waveguides Application and Development). We thank Mauro Paternostro, Maria Antonietta Ricci, Beatrice Polacchi, Camilla Sarra, and Federico Pegoraro for useful help and discussions.

*andrea.geraldi@uniroma1.it

[1] G. S. Engel, T. R. Calhoun, E. L. Read, T.-K. Ahn, T. Mančal, Y.-C. Cheng, R. E. Blankenship, and G. R. Fleming, Evidence for wavelike energy transfer through quantum coherence in photosynthetic systems, Nature (London) 446, 782 (2007).

[2] N. Lambert, Y.-N. Chen, Y.-C. Cheng, C.-M. Li, G.-Y. Chen, and F. Nori, Quantum biology, Nat. Phys. 9, 10 (2013).

[3] M. Mohseni, P. Rebentrost, S. Lloyd, and A. Aspuru-Guzik, Environment-assisted quantum walks in photosynthetic energy transfer, J. Chem. Phys. 129, 174106 (2008).

[4] M. B. Plenio and S. F. Huelga, Dephasing-assisted transport: Quantum networks and biomolecules, New J. Phys. 10, 113019 (2008).

[5] V. B. Bulchandani, C. Karrasch, and J. E. Moore, Superdiffusive transport of energy in generic Luttinger liquids, arXiv:1904.09287.

[6] H. van Beijeren, Exact Results for Anomalous Transport in One-Dimensional Hamiltonian Systems, Phys. Rev. Lett. 108, 180601 (2012).

[7] E. Kim, A. J. Martínez, S. E. Phenisee, P. Kevrekidis, M. A. Porter, and J. Yang, Direct measurement of superdiffusive energy transport in disordered granular chains, Nat. Commun. 9, 640 (2018).

[8] R. Vasseur and J. E. Moore, Nonequilibrium quantum dynamics and transport: From integrability to many-body localization, J. Stat. Mech. (2016) 064010.

[9] S. Hoyer, M. Sarovar, and K. B. Whaley, Limits of quantum speedup in photosynthetic light harvesting, New J. Phys. 12, 065041 (2010).

[10] P. W. Anderson, Absence of diffusion in certain random lattices, Phys. Rev. 109, 1492 (1958).

[11] M. Segev, Y. Silberberg, and D. N. Christodoulides, Anderson localization of light, Nat. Photonics 7, 197 (2013).

[12] T. Schwartz, G. Bartal, S. Fishman, and M. Segev, Transport and Anderson localization in disordered two-dimensional photonic lattices, Nature (London) 446, 52 (2007).

[13] A. Crespi, R. Osellame, R. Ramponi, V. Giovannetti, R. Fazio, L. Sansoni, F. De Nicola, F. Sciarrino, and P. Mataloni, Anderson localization of entangled photons in an integrated quantum walk, Nat. Photonics 7, 322 (2013).

[14] L. Levi, Y. Krivolapov, S. Fishman, and M. Segev, Hypertransport of light and stochastic acceleration by evolving disorder, Nat. Phys. 8, 912 (2012).

[15] A. Schreiber, K. N. Cassemiro, V. Potoček, A. Gábris, I. Jex, and C. Silberhorn, Decoherence and Disorder in Quantum Walks: From Ballistic Spread to Localization, Phys. Rev. Lett. 106, 180403 (2011).

[16] T. A. Brun, H. A. Carteret, and A. Ambainis, Quantum to Classical Transition for Random Walks, Phys. Rev. Lett. 91, 130602 (2003).

[17] M. A. Broome, A. Fedrizzi, B. P. Lanyon, I. Kassal, A. Aspuru-Guzik, and A. G. White, Discrete Single-Photon Quantum Walks with Tunable Decoherence, Phys. Rev. Lett. 104, 153602 (2010).

[18] S. E. Venegas-Andraca, Quantum walks: A comprehensive review, Quantum Inf. Process. 11, 1015 (2012).

[19] J. Kempe, Quantum random walks: An introductory overview, Contemp. Phys. 44, 307 (2003).

[20] A. Ahlbrecht, H. Vogts, A. H. Werner, and R. F. Werner, Asymptotic evolution of quantum walks with random coin, J. Math. Phys. 52, 042201 (2011).

[21] B. Do, M. L. Stohler, S. Balasubramanian, D. S. Elliott, C. Eash, E. Fischbach, M. A. Fischbach, A. Mills, and B. Zwickl, Experimental realization of a quantum quincunx by use of linear optical elements, J. Opt. Soc. Am. B 22, 499 (2005).

[22] T. Nitsche, F. Elster, J. Novotný, A. Gábris, I. Jex, S. Barkhofen, and C. Silberhorn, Quantum walks with dynamical control: Graph engineering, initial state preparation and state transfer, New J. Phys. 18, 063017 (2016).

[23] F. Flamini, N. Spagnolo, and F. Sciarrino, Photonic quantum information processing: A review, Rep. Prog. Phys. 82, 016001 (2019).

[24] L. Sansoni, F. Sciarrino, G. Vallone, P. Mataloni, A. Crespi, R. Ramponi, and R. Osellame, Two-Particle BosonicFermionic Quantum Walk via Integrated Photonics, Phys. Rev. Lett. 108, 010502 (2012).

[25] Á. Cuevas, A. Geraldi, C. Liorni, L. D. Bonavena, A. De Pasquale, F. Sciarrino, V. Giovannetti, and P. Mataloni, All-optical implementation of collision-based evolutions of open quantum systems, Sci. Rep. 9, 3205 (2019).

[26] See Supplemental Material at http://link.aps.org/ supplemental/10.1103/PhysRevLett.123.140501 for more details regarding the experimental setup and the experimental results with single and two-photon states.

[27] A. Geraldi, L. D. Bonavena, C. Liorni, P. Mataloni, and Á. Cuevas, A novel bulk-optics scheme for quantum walk with high phase stability, Condens. Matter 4, 14 (2019).

[28] S. Havlin and D. Ben-Avraham, Diffusion in disordered media, Adv. Phys. 51, 187 (2002). 